\title{
Diverse manifestations and outcomes in two COVID-19 cases with concomitant leukemia
}

\author{
Hongbo Chen ${ }^{1}$, Hui $\mathrm{Li}^{2}$, Yining Qiu ${ }^{1}$, Runming $\mathrm{Jin}^{1}$, and Xiaoyan $\mathrm{Wu}^{1,3}$ \\ ${ }^{1}$ Wuhan Union Hospital \\ ${ }^{2}$ Wuhan Childrens Hospital \\ ${ }^{3}$ University Hospital
}

August 28, 2020

Title: Diverse manifestations and outcomes in two COVID-19 cases with concomitant leukemia

Authors: Hongbo Chen ${ }^{1}$, Hui $\mathrm{Li}^{2}$, Yining Qiu ${ }^{1}$, Runming $\mathrm{Jin}^{1}$, Xiaoyan $\mathrm{Wu}^{1}$

Affiliations:

1 Department of Pediatrics, Union Hospital, Tongji Medical College, Huazhong University of Science and Technology, Wuhan, China

2 Department of Oncology, Wuhan Children's Hospital, Tongji Medical College, Huazhong University of Science and Technology, Wuhan, China

Correspondence:

Xiaoyan Wu, MD, PhD. Address: Department of Pediatrics, Union Hospital, Tongji Medical College, Huazhong University of Science and Technology, 1277 Jiefang Avenue, Wuhan, 430022, Hubei, China. Email: xwu@hust.edu.cn.

Text word count: 666

One figure contained.

Running title: COVID-19 in pediatric leukemia patients

Keyword: pediatric leukemia; COVID-19; SARS-CoV-2

To the editor,

Several studies have documented Severe acute respiratory syndrome corona virus 2 (SARS-CoV-2) infection in children, while the infection in children with cancer is rarely reported. [1-4] Here we present two cases of COVID-19 in pediatric leukemia patients.

The first case was a four-year-old boy with preceding acute lymphoblastic leukemia. He was at the stage one of continuation phase. His mother was a suspected case of COVID-19 characterized by fever, cough, a viral pneumonia in the thoracic CT scan and a negative nucleic acid result for SARS-CoV-2. They lived in the same house and had a close contact. He received a nucleic acid test for SARS-CoV-2 before admission for the chemotherapy. Both nasopharyngeal swab and anus swab tested positive. The patient had no fever, cough, diarrhea or any other concerning symptoms. The complete blood count revealed white blood cell $3.43 \times 10^{6} / \mathrm{mL}$, neutrophil $35.2 \%$, lymphocyte $55.1 \%$, erythrocyte $3.94 \times 10^{9} / \mathrm{mL}$, hemoglobin $11.5 \mathrm{~g} / \mathrm{dL}$, platelet $287 \times 10^{6} / \mathrm{mL}$. And the thoracic CT scan found patchy opacity in the upper segment of left lower 
lobe. A diagnosis of COVID-19 was confirmed and he was then transferred to a designated hospital for further treatment.

After admitted to the designated hospital, he got a comprehensive examination. No positive finding was identified after the physical examination. And the tests for serum D-dimer, ferritin, cytokines (interleukin-6, tumor necrosis factor- $\alpha$, interferon- $\gamma$ ) returned normal. He received a continuous treatment of interferon $\alpha$ $-1 b$ intravenously and nebulization for eight days. Three days after admission, the nucleic acid test turned negative and was consistently negative two days later. A repeated thoracic CT scan showed an apparent recovery. And he was discharged eight days after admission and isolated at home for another 14 days.

The second case was an eight-year-old boy with acute lymphoblastic leukemia. He was at the maintenance stage of the chemotherapy. He was a resident of Wuhan city, which was the epicenter of COVID-19 outbreak, even though he had no obvious history of close contact with suspected or confirmed COVID-19 cases. He developed fever three days (D3) after admission for chemotherapy. D-dimer was normal, while interleukin-6 (IL-6), C reactive protein (CRP) and procalcitonin (PCT) were elevated. The thoracic CT scan revealed bilateral pneumonia (Figure 1A). And the he was tested positive for SARS-CoV-2 by RT-PCR.

He was then given ribavirin, arbidol, antibiotics, methylprednisolone, immunoglobulins and other supporting care. However, the fever persisted and he was transferred to the intensive care unit with the invasive ventilation for respiratory failure on D24. And the thoracic CT scan demonstrated an aggressive progress (Figure 1B). Thus, convalescent plasma from recovered COVID-19 patients was transfused to the boy patient every five days for three times. The temperature turned to normal on D44, and the invasive ventilation was withdrawn a week later. But the patient got fever and tachypnea again on D58, and the blood culture revealed multidrug-resistant Acinetobacter baumannii. He was supported with invasive ventilation till finally abandon by the parents for persistent coma.

The two cases showed a completely different manifestations and outcomes in pediatric leukemia patients after infected with SARS-CoV-2. Although patients with leukemia have compromised immunity, they may present mild symptoms or even no symptoms and survive the COVID-19. Treatment for children with COVID-19 is diverse but lack of strong evidence. Most therapies are empirical. For patient 1, the intravenous and nebulization of interferon $\alpha-1 b$ seemed effective and cured the COVID-19 pneumonia. For patient 2, antiviral drugs (ribavirin, arbidol, interferon $\alpha-1 b$ ) combined with steroids, immunoglobins and antibiotics did not take effect and the condition deteriorated. Latter transfusion of convalescent plasma from recovered COVID-19 patients turned the scale. The fever was extinguished and the invasive ventilation withdrew. However, secondary infection of multidrug-resistant Acinetobacter baumannii aggravated the situation and lead to multi-organ failure and irreversible brain injury, though the thoracic CT scan lessened (Figure 1C).

The clinical manifestations and outcomes of COVID-19 in pediatric leukemia patients are diverse but sometimes lethal especially concomitant of bacterial or fungal infections. It is critical for timely recognition and treatment of COVID-19 in this special population.

Author's contributions: Hongbo Chen, Hui Li and Yining Qiu wrote the manuscript. They contributed equally as the co-first authors. Runming Jin and Xiaoyan Wu designed the study and finalized the manuscript.

Conflict of interests: All authors declared no competing interests.

Funding: Xiaoyan Wu is supported by an innovative research grant from Wuhan Science and Technology Department (No. 2019020701011503).

This work was approved by the ethics committee of Union Hospital, Tongji Medical College, Huazhong University of Science and Technology.

References

1. Dong Y, Mo X, Hu Y, et al. Epidemiology of COVID-19 among children in China. Pediatrics . 2020; 145(6):e20200702. 
2. Stokes C, Sabnis H, Yildirim I, et al. Severe COVID-19 disease in two pediatric oncology patients. Pediatr Blood Cancer . 2020; 67:e28432.

3. Schied A, Trovillion E and Moodley A. SARS-CoV-2 infection in a neutropenic pediatric patient with leukemia: Addressing the need for universal guidelines for treatment of SARS-CoV-2-positive, immunocompromised patients. Pediatr Blood Cancer . 2020; 67:e28546.

4. Boulad F, Kamboj M, Bouvier N, et al. COVID-19 in children with cancer in New York City. JAMA Oncol . 2020; e202028.

Figure legend

Figure 1. Thoracic CT scans of the patient 2.

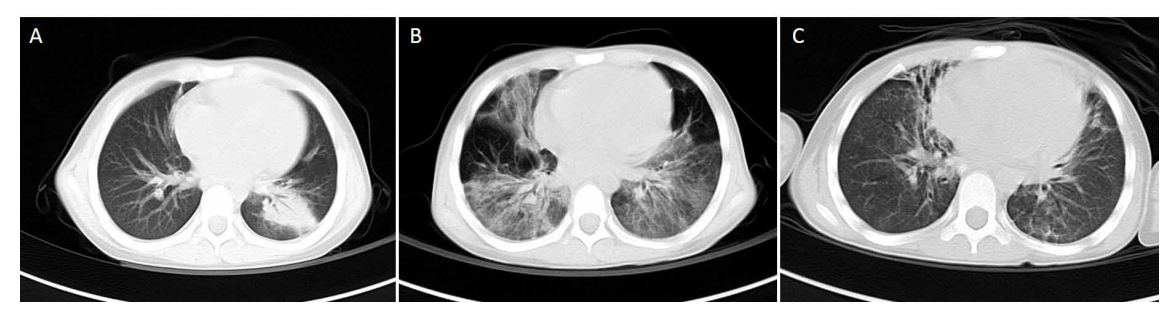

